\title{
Third JUAMI connects US and African Fellows around sustainable energy materials in Uganda
}

\author{
By Carlos Biaou and Julie Fornaciari
}

$\mathrm{T}$ he Joint Undertaking for an African Materials Institute (JUAMI) embarked on its third school in Kampala, Uganda, this past December. For 13 days, scholars from across the United States and Africa came together to learn about sustainable energy materials, propose their own solutions to the problems, and engage in cultural exchange - both scientific and social.

The program was developed in 2012 to provide opportunities for collaboration between American and East African scholars. This third school follows previous ones that took place in Arusha, Tanzania (2016) and Addis Ababa, Ethiopia (2012). The JUAMI community continues to grow, and this year's gender-balanced cohort was made up of 62 Fellows: 24 from the United States, 1 from Israel, and 37 from 10 different African countries.

JUAMI incorporated many program components within the two weeks: a poster session; tutorials, which were paired with hands-on laboratories; research lectures designed to leverage the learning from the tutorials; and a plenary lecture aimed at providing a broader context for JUAMI activities. The topics covered spanned from photovoltaics to flow cells to thermoelectrics, all focused on materials research being done in the area.

The poster session featured research projects from all of the participants. Poster topics ranged from studies of polymer solar cells to investigations of structural and electronic properties of crystal structures, including $\mathrm{Na}_{2} \mathrm{Ti}_{3} \mathrm{O}_{7}$ and $\mathrm{H}_{2} \mathrm{Ti}_{3} \mathrm{O}_{7}$. With explicit instructions to include information about what expertise and capabilities participants could offer and what they were seeking, discussions about potential collaborations were encouraged.

The seven tutorials, delivered by an international cohort of professors, covered the topics of (1) conducting polymers (Jiaxing Huang, Northwestern University); (2) batteries (Adrian Hightower, Metropolitan Water District of Southern California); (3) photovoltaics (David Cahen, Weizmann Institute); (4) flow cells and fuel cells (S.R. Narayan, University of Southern California); (5) nanomaterials (Sara Skrabalak, Indiana University Bloomington); (6) electrolysis (Thomas Mallouk, The Pennsylvania State University); and (7) thermoelectrics (G. Jeffrey Snyder, Northwestern University). The associated laboratory activities gave students the opportunity to work together and have fun with science. The laboratories spanned from using an Arduino board to power a battery, to running a methanol fuel cell, to making nanoparticles using tea extract. Joshua Santana, a chemistry $\mathrm{PhD}$ candidate at Indiana University Bloomington, who served as the teaching assistant for the nanoparticle laboratory, noted that "many East African participants in the JUAMI program have not had the same access to laboratory equipment throughout their educational career." Therefore, each experiment was developed to be able to bring as little equipment as possible from abroad and use resources available in Africa.

Leading international researchers in the field delivered the lectures. These included Linda Nazar (University of Waterloo), Luisa Whittaker-Brooks (The University of Utah), and Vladan Stevanovic (National Renewable Energy Laboratory), who spoke about the latest advances in their respective laboratories in battery research, hybrid perovskite photovoltaics, and computational prediction of new thermoelectrics. Speakers from Africa, including Mmantsae Diale (University of Pretoria), Kiros Guesh (Aksum University, JUAMI 2012 alumnus), and Justus Simiyu (University of Nairobi, tentative local host for JUAMI 2020) detailed advances made in their laboratories, despite limited resources.

Carlos Biaou and Julie Fornaciari, University of California, Berkeley 
Overall, the lectures and tutorials provided insights into fields that were either closely or directly related to the areas of study of JUAMI scholars. A highlight of the two-week school was a plenary talk by Sir David King, senior strategy advisor to the president of Rwanda. During his lecture and ensuing Q\&A session, King shared his thoughts on the upcoming challenges in climate change around the world. His frank assessment inspired action rather than caused despair.

A central theme of JUAMI is the creation of long-term international partnerships. As such, scholars formed groups, each composed of a mix of Fellows from different countries, and designed projects focused on education, outreach, and scientific research.

On the education front, one project aims to build a virtual computing facility to enable access to state-of-the-art software along with high computing speed by hosting the service on a cloud provider. The project is currently in the second round of its fundraising phase and was selected as a finalist for receiving a grant from the University of California, Berkeley. Timothy Tibesigwa, a $\mathrm{PhD}$ student in mechanical engineering at Makerere University in Uganda, plans on using the facility for life-cycle assessments "to compare the environmental impacts of using biodiesel from locally sourced feedstocks to petroleum diesel for transportation."

On the outreach front, a project aspires to mentor female students in rural East Africa to pursue STEM subjects for their future careers. The team plans on achieving that goal by targeting primary and secondary schools most in need and connecting them with the appropriate mentors from local universities (juamimentors.wixsite.com/stem).

On the scientific front, some Fellows have decided to engage in an ambitious cross-continental project for the synthesis and characterization of conjugated polymer solar cells. The plan is to perform density functional theory calculations in Sudan, polymer synthesis in Ethiopia, and solar-cell fabrication and characterization in Germany, Colorado, and Tennessee. The collaboration is ongoing, as polymers
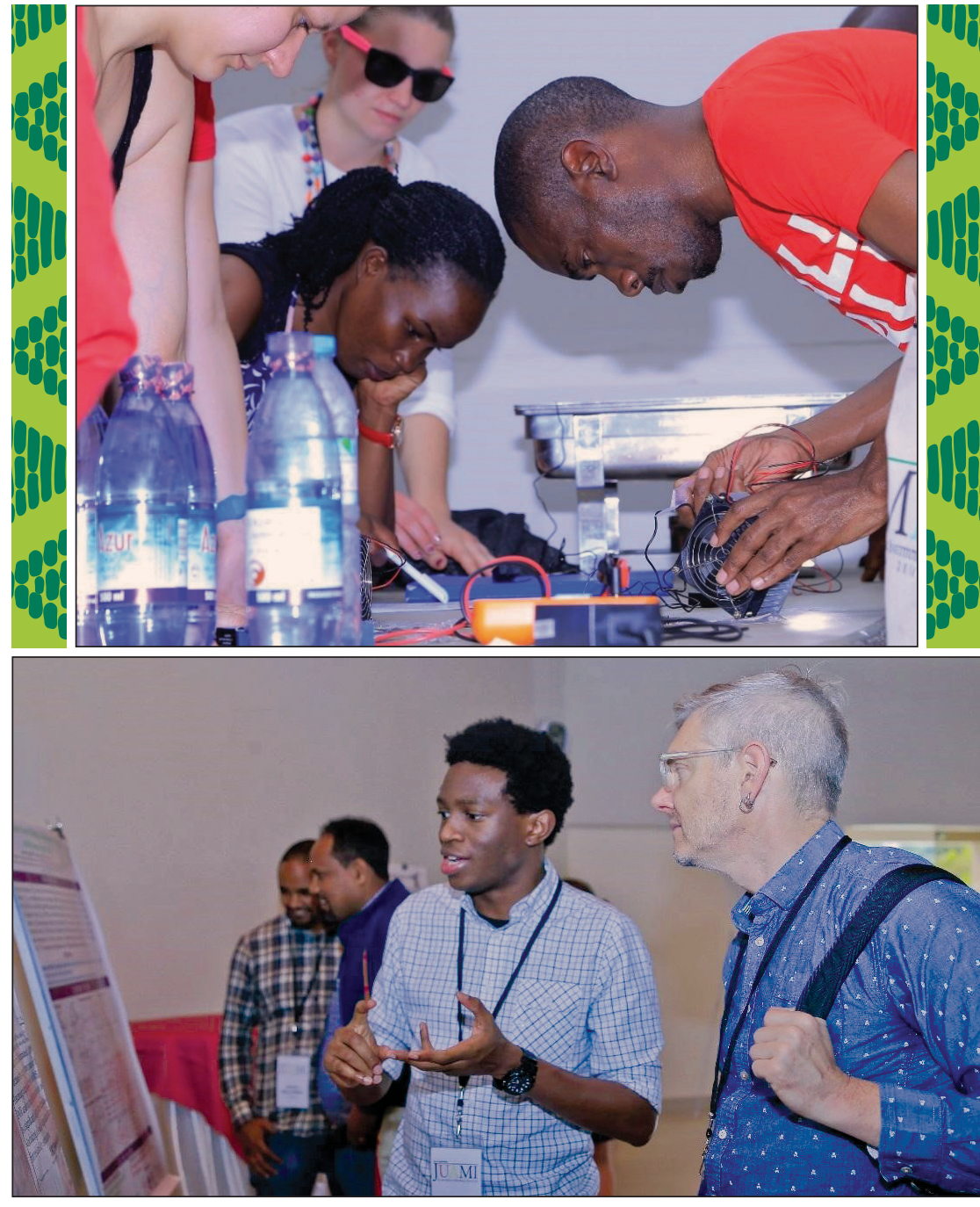

(Top) JUAMI Fellows explore thermoelectricity in the last hands-on activity of the program; (Bottom) JUAMI Fellows engage with fellow scholars and professors at the kick-off poster session.

were synthesized and shipped to Germany for the next processing phase.

Beyond the rigorous schedule that kept the JUAMI Fellows busy throughout the two weeks, there was time to explore the beauty and culture of Uganda. The encouragement to collaborate to address societal problems through technical solutions remained in the forefront. In particular, at the conference banquet, the featured speaker was the Minister of Works and Transport, the Honorable Monica Azuba Ntege. Leaning on her engineering background, she exhorted the fellows to tackle challenging scientific issues, beyond those addressed by JUAMI. Throughout the two weeks, the community gained through the instructional and social aspects of the program, and these now serve as the building blocks to partnerships and friendships that are outliving the program.

JUAMI 2018 was funded primarily by a grant from the US National Science Foundation (NSF) proposed by Sossina M. Haile (Northwestern University), Simon Billinge (Columbia University), and Thomas Mallouk (The Pennsylvania State University). The 2018 institute was co-hosted by Makerere University in Kampala, Uganda. The local organizing committee was led by Ireeta Winston (Makerere University) and MargaretAnne (Mazzi) Wampamba (NSF, retired), who worked with US organizers to select student participants and develop the twoweek curriculum. 